\title{
Laser Resonance Ionization Spectroscopy on Lutetium for the MEDICIS Project
}

\author{
V. Gadelshin ${ }^{1,4}$ • T. Cocolios ${ }^{2}$ • V. Fedoseev ${ }^{3}$ • R. Heinke ${ }^{1}$ • T. Kieck ${ }^{1}$ • \\ B. Marsh $^{3}$ • P Naubereit ${ }^{1} \bullet$ S. Rothe ${ }^{3,5}$ • T. Stora ${ }^{3}$ • D. Studer ${ }^{1} \bullet$ \\ P. Van Duppen ${ }^{2} \cdot K$. Wendt ${ }^{1}$
}

\begin{abstract}
The MEDICIS-PROMED Innovative Training Network under the Horizon 2020 EU program aims to establish a network of early stage researchers, involving scientific exchange and active cooperation between leading European research institutions, universities, hospitals, and industry. Primary scientific goal is the purpose of providing and testing novel radioisotopes for nuclear medical imaging and radionuclide therapy. Within a closely linked project at CERN, a dedicated electromagnetic mass separator system is presently under installation for production of innovative radiopharmaceutical isotopes at the new CERN-MEDICIS laboratory, directly adjacent to the existing CERN-ISOLDE radioactive ion beam facility. It is planned to implement a resonance ionization laser ion source (RILIS) to ensure high efficiency and unrivaled purity in the production of radioactive ions. To provide a highly efficient ionization process, identification and characterization of a specific multi-step laser ionization scheme for each individual element with isotopes of interest is required. The element lutetium is of primary relevance, and therefore was considered as first candidate. Three two-step excitation schemes for lutetium atoms are presented in this work, and spectroscopic results are compared with data of other authors.
\end{abstract}

Keywords. CERN-MEDICIS • lutetium • isotope separation • laser ionization spectroscopy.

PACS. 28.60.ts; 29.30.-h; 32.10.Bi; 32.30.-r; 32.80.-t; 42.62.Fi; 87.19.xj

\footnotetext{
${ }^{1}$ Institute of Physics, University of Mainz, Germany

${ }^{2}$ Institute for Nuclear and Radiation Physics, KU Leuven, Belgium

${ }^{3}$ EN Department, CERN, Geneva, Switzerland

${ }^{4}$ Faculty of Physics and Technology, Ural Federal University, Yekaterinburg, Russia

${ }^{5}$ The University of Manchester, Manchester, United Kingdom

E-mail: gadelshin@uni-mainz.de
} 


\section{Introduction}

The MEDICIS project aims for the production, development, and application of novel medical radioisotopes for radiotherapy, functional imaging and comparable techniques. The project is carried out in a broad collaboration of different European scientific, medical, and business institutions, and will involve the future CERN-MEDICIS facility for medical radioisotope production [1]. According to the goals, the project is divided into three parts called Work Packages (WPs), which are related basically to the fields of Isotope Production (WP1), Functional Imaging (WP2), and Radiotherapy (WP3). Within WP1 University of Mainz has taken over responsibilities for development and design of a suitable laser system for the project, which is to be installed at the CERN MEDICIS facility to implement resonance ionization.

There are three nuclear reaction types for the production of radioisotopes: fission, spallation and fusion, induced by bombardment of a target with 1.4 $\mathrm{GeV}$ proton beam. From the multitude of different isotopes generated in the target unit, the desired medical radioisotopes must be extracted with highest purity and efficiency. For this purpose, the resonance ionization laser ion source (RILIS) technology combined with a conventional electromagnetic separating unit will be used. In the RILIS method, only the desired element will be ionized through a multi-step resonant excitation/ionization process by well-tuned laser photons [2]. Thus, selected atoms will be driven through one or two intermediate excited state into an auto-ionizing state (AI), leading to high ionization probability [3], for the subsequent isotope separation of ionized atoms.

The task addressed here lies in the development of an excitation scheme for each desired element to provide the most efficient ionization process well suitable for the MEDICIS project. In the work presented here, several possible two-step excitation schemes of the rare earth element lutetium, which is of high relevance for the MEDICIS project, were investigated, as presented in Figure 1.

\section{Lutetium spectroscopy survey}

Today lutetium-177 is one of the most demanded medical radioisotope in the world. Its application in the field of so-called theranostics provides safe and powerful treatment with specific attractive features involving application of both - soft beta radiation as well as the low-energy gammaemission, the possibility of reliable labeling of biomolecules, and palliation effects induced by the radiotherapy $[4,5]$.

Laser ionization spectroscopy on lutetium atoms was performed already decades ago with dye laser systems. First results were obtained by Soviet [6], and US researchers [7] using a blue-blue two-step excitation scheme (scheme D on the Fig. 1). 


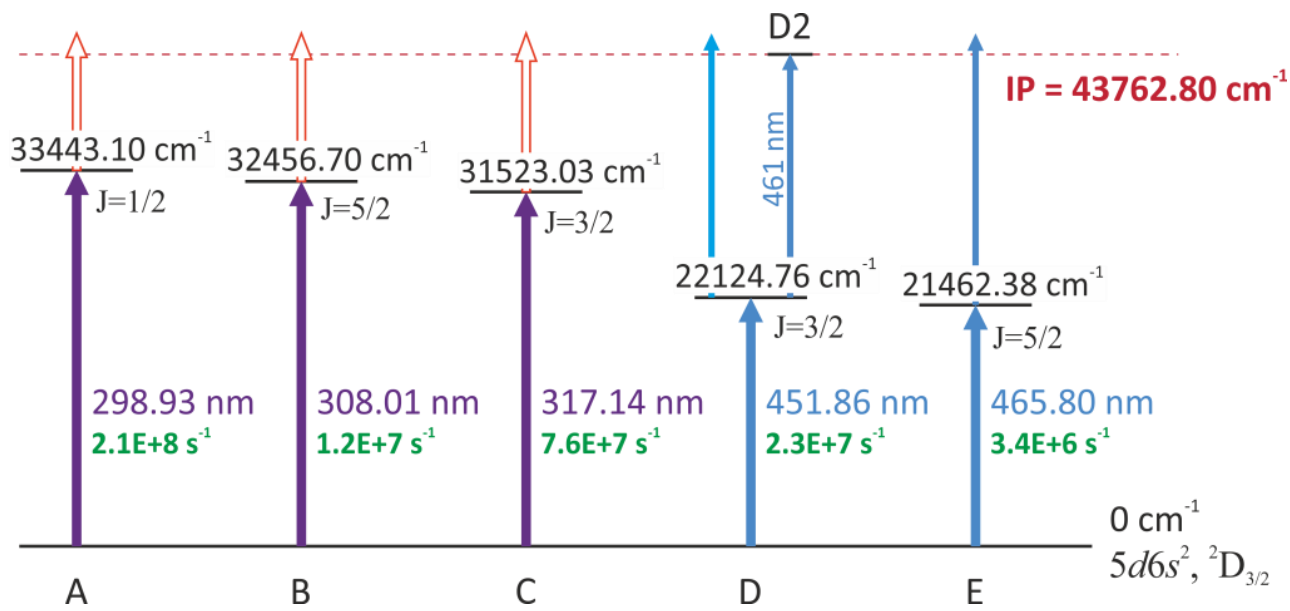

Fig. 1. Two-step excitation schemes of lutetium accessible to tunable Titanium: sapphire lasers. For each first excitation step, transition strength, wavelength, upper level energy, and $\mathrm{J}$ value are given. Schemes A to $\mathrm{C}$ have been studied in this work, schemes $\mathrm{D}$ in $[6,7,8]$ and scheme $\mathrm{E}$ in [9]. IP - ionization potential.

The most efficient transition found by them, denoted as the scheme D2 in Fig. 1, was used in a research work for geological analytics [8]. More accurate spectroscopic analysis of lutetium auto-ionizing states was carried out in [9] using several different first steps (schemes D, E and others). Presently, there are activities on the set up of a separating unit for lutetium using the atomic vapor laser isotope separation technology (AVLIS), being developed in Russia and using a dye laser system [10, 11].

In the framework of the MEDICIS project, it is foreseen to exclusively use highly reliable and almost completely maintenance free tunable pulsed Titanium:sapphire lasers as main components of the laser ion source. However, many of the existing dye laser ionization schemes are not applicable for Titanium:sapphire lasers. Correspondingly, spectroscopic investigations on the development of an ionization scheme for lutetium based exclusively on Titanium:sapphire laser radiation were started at Mainz University in parallel to activities at the TRIUMF facility in Canada, where scheme D of Fig. 1 has been studied in detail.

\section{Experimental setup}

Titanium:sapphire laser based resonance ionization spectroscopy on lutetium atoms has been performed at University of Mainz on an experimental unit named the Mainz Atomic Beam Unit (MABU). A sketch of the device is presented in Figure 2. 


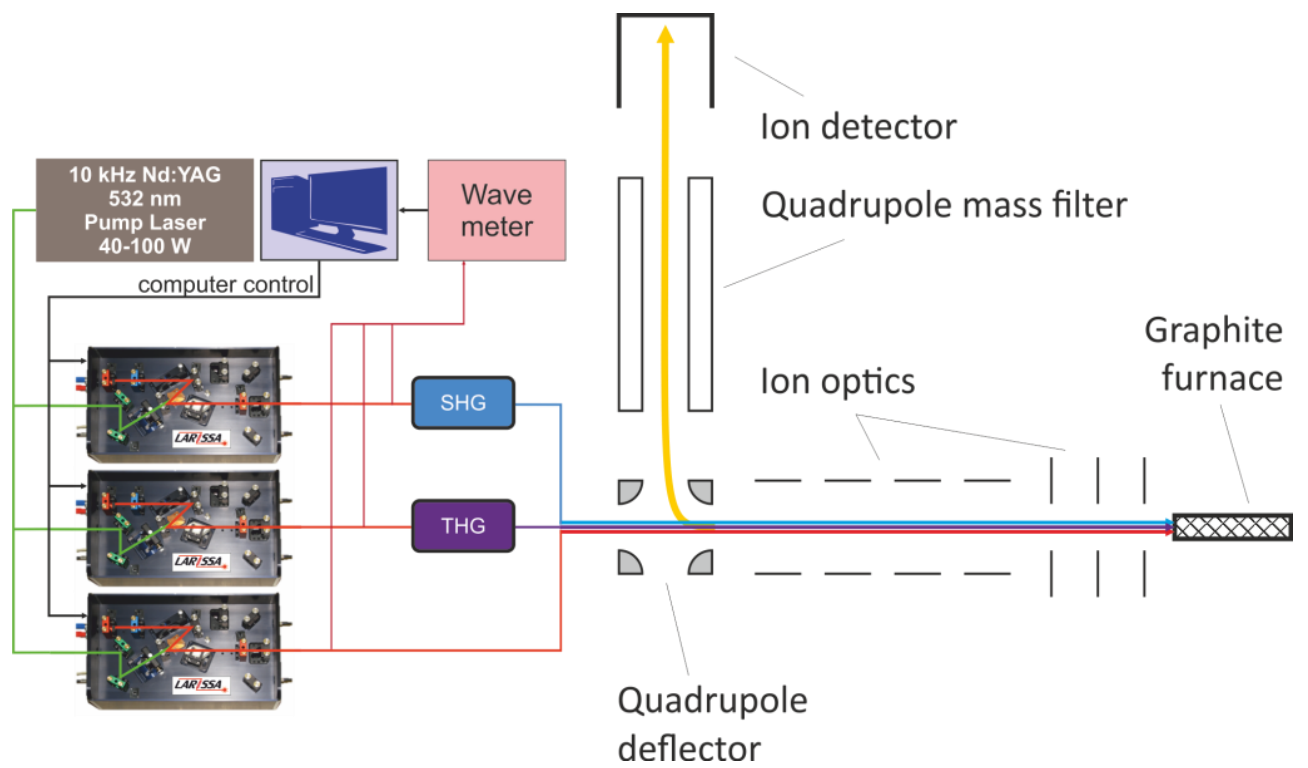

Fig. 2. Schematic drawing of the Mainz Atomic Beam Unit (MABU) and the Titanium:sapphire laser system used. SHG and THG denote second and third harmonic generators, respectively. The wavelength of the lasers is monitored by a wave meter, which is directly connected to the data acquisition and provides automated laser scanning.

It consists of a resistively heated graphite furnace, providing atomization of lutetium at $\approx 1700^{\circ} \mathrm{C}$. A set of ion optics permits acceleration and beam shaping of the ions produced by laser radiation. With an electrostatic quadrupole deflector, the ion beam is isolated from neutral background. A radio-frequency quadrupole mass filter is applied for selection of a particular mass. Finally, a channel electron multiplier detector provides quantitative ion registration on a single ion level (see Fig. 2) [12].

The MABU is combined with a full Titanium:sapphire laser system for resonant multi-step ionization. The system consists of two to three tunable high repetition rate pulsed Titanium:sapphire lasers of the Mainz type, pumped jointly by a commercial Nd:YAG laser at $532 \mathrm{~nm}$ with a repetition rate of $10 \mathrm{kHz}$ and $60 \mathrm{~W}$ output power. The fundamental wavelength range of the Titanium:sapphire lasers extends from 690 to $960 \mathrm{~nm}$, with up to $5 \mathrm{~W}$ output power and a spectral line width of about $5 \mathrm{GHz}$. Via frequency doubling or tripling, wavelengths in the blue and UV spectral range are also accessible. One of the lasers is equipped with a motorized diffraction grating for frequency selection, which permits to perform a continuous wavelength scan across the entire amplification range [13].

Initial accomplishment of laser resonance ionization of lutetium involving auto-ionizing states is the starting point for refined investigations in spectroscopy and for studies on ionization efficiencies. 


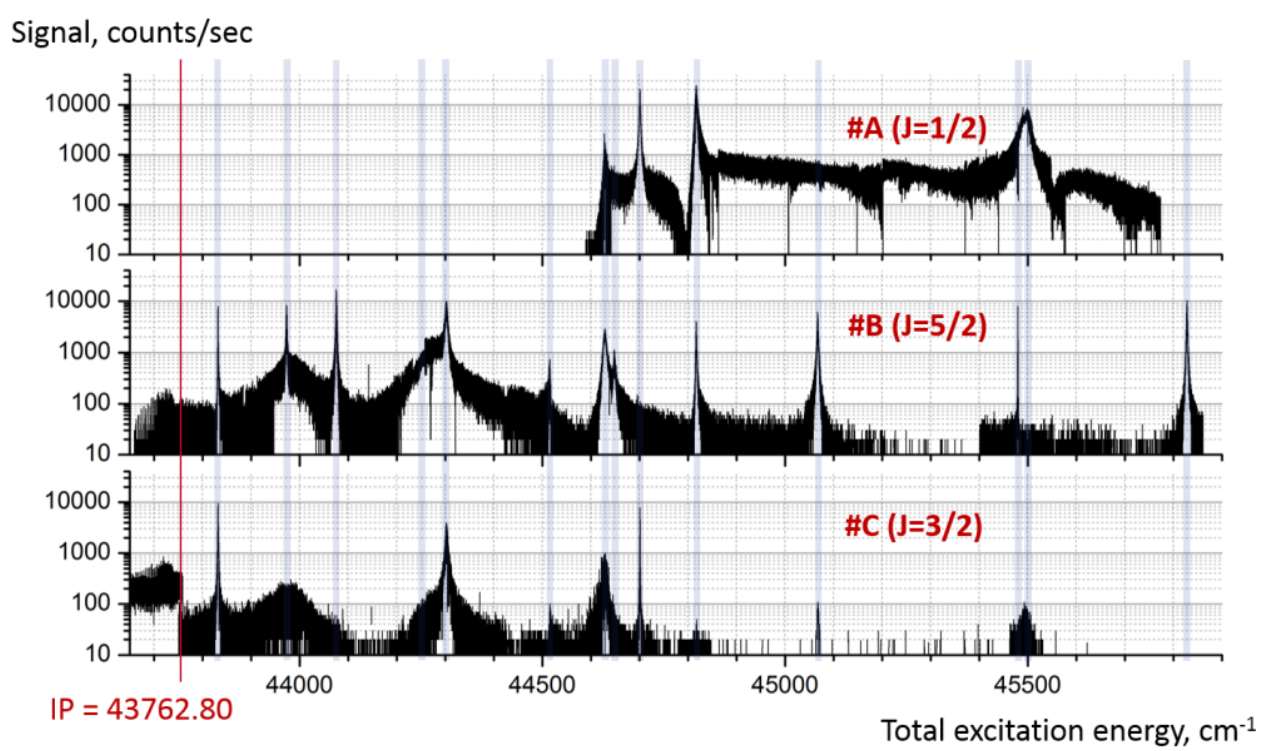

Fig. 3. Observed photoionization spectra of lutetium for the two-step excitation schemes $\mathrm{A}, \mathrm{B}$, and $\mathrm{C}$ above the ionization potential (IP); grey vertical lines highlight the position of auto-ionizing states (AIs) in the spectra.

Identification of a suitable and efficient excitation scheme is crucial for the production of medical radioisotopes at CERN-MEDICIS to meet the stringent standards of nuclear medicine products. From detailed analysis of databases on atomic spectra $[14,15]$, several possible transitions for the first excitation step were chosen (schemes A-E on the Fig. 1), which are accessible with Titanium:sapphire lasers. All first steps of the selected schemes exhibit promising transition strengths and should thus provide a powerful excitation process. Lutetium samples were prepared from a standard solution of lutetium (III) oxide in 5\% nitric acid with concentration of lutetium of about $1000 \mu \mathrm{g} / \mathrm{ml}$, purchased from Alfa Aesar [16]. A drop of about 10 to $100 \mu \mathrm{l}$, containing some 10 to $100 \mu \mathrm{g}$ of lutetium, was placed on a small piece of zirconium foil, which serves as carrier and reducing agent, dried and finally inserted in the graphite furnace.

\section{Results}

Extensive laser ionization spectroscopy on lutetium atoms was carried out in the two-step UV-red excitation schemes A, B, and C of Fig. 1 for the first time. The investigation of these schemes was performed with the widerange tunable Titanium:sapphire laser system. A compilation of spectra of autoionizing resonances of lutetium in these schemes just above the IP are presented in Figure 3. 
Table 1. Energetic positions of auto-ionizing states (AIs) of lutetium observed in the different excitation schemes A to E of Fig. 1. Relative intensity are indicated by the letters $(\mathrm{H}, \mathrm{M}, \mathrm{L})$ representing high, middle and low transition rate, respectively; or $(0)$ in the case, when autoionization has not occurred. For each schemes the $J$ value of the first excited state is given in the first row. In the last column, the assigned $J_{A I}$ value for the observed AIs is presented. Scheme D is divided by «slash» symbol to refer to the results from [7] or [6,9], respectively. The data corresponding to scheme $\mathrm{E}$ was taken from [9].

\begin{tabular}{|l|c|c|c|c|c|c|}
\hline $\begin{array}{l}\text { Energy, } \\
\text { cm-1 }\end{array}$ & \#A & \#B & \#C & \#D & \#E & $J_{A I}$ \\
\hline 43831.0 & - & $\mathrm{M}$ & $\mathrm{H}$ & $\mathrm{H} / \mathrm{H}$ & $\mathrm{L}$ & $3 / 2,5 / 2$ \\
\hline 43972.7 & - & $\mathrm{M}$ & $\mathrm{L}$ & $\mathrm{L} / 0$ & $\mathrm{~L}$ & $3 / 2,5 / 2$ \\
\hline 44074.9 & - & $\mathrm{H}$ & 0 & $0 / 0$ & $\mathrm{H}$ & $7 / 2$ \\
\hline 44249.3 & - & 0 & 0 & $\mathrm{M} / 0$ & 0 & $1 / 2,3 / 2,5 / 2$ \\
\hline 44301.6 & - & $\mathrm{H}$ & $\mathrm{M}$ & $\mathrm{H} / \mathrm{M}$ & $\mathrm{L}$ & $3 / 2,5 / 2$ \\
\hline 44514.6 & - & $\mathrm{L}$ & $\mathrm{L}$ & $\mathrm{M}^{* * / \mathrm{L}}$ & $\mathrm{M}$ & $3 / 2,5 / 2$ \\
\hline 44628.5 & $\mathrm{M}$ & $\mathrm{M}$ & $\mathrm{L}$ & $\mathrm{M}^{* * / \mathrm{L}}$ & $\mathrm{L}$ & $3 / 2$ \\
\hline 44647.1 & 0 & $\mathrm{~L}$ & 0 & $0 / 0$ & $\mathrm{M}$ & $7 / 2$ \\
\hline 44699.7 & $\mathrm{H}$ & 0 & $\mathrm{H}$ & $\mathrm{M} * * / \mathrm{M}$ & 0 & $1 / 2$ \\
\hline 44816.9 & $\mathrm{H}$ & $\mathrm{M}$ & $\mathrm{L}$ & $0 / \mathrm{L}$ & $\mathrm{L}$ & $3 / 2$ \\
\hline 45066.9 & 0 & $\mathrm{M}$ & $\mathrm{L}$ & $-/ \mathrm{L}$ & - & $5 / 2$ \\
\hline 45478.3 & 0 & $\mathrm{M}$ & 0 & - & - & $7 / 2$ \\
\hline 45496.1 & $\mathrm{M}$ & 0 & $\mathrm{~L}$ & - & - & $1 / 2$ \\
\hline 45827.5 & - & $\mathrm{H}$ & - & - & - & $3 / 2,5 / 2,7 / 2$ \\
\hline
\end{tabular}

(-) means that a probable AI lies out of the measurement range;

$(*)$ - detection of the transition to the AI state is under discussion;

$(* *)$ - in the original paper, authors did not relate the peak to an AI.

A multitude of different auto-ionizing states have been reconfirmed together with the observation of one newly discovered and located at the total excitation energy of 45496,1 $\mathrm{cm}^{-1}$. A comparison between relative intensities of the photoionization signal observed in this work and data from dye laser ionization spectroscopy of other authors for the blue-blue schemes $\mathrm{D}$ and $\mathrm{E}$ is presented in Table 1.

The obtained spectra permit to select an efficient two-step ionization scheme for lutetium atoms. For this purpose, a strong transition into an autoionizing state together with high transition strength of the first excitation step is required. The most promising candidates are transitions to the two AIs located at $44699,7 \mathrm{~cm}^{-1}$ or at $44816,9 \mathrm{~cm}^{-1}$ in scheme A for UV-red excitation schemes (compare the upper trace in Fig. 3), and to AIs located at 43831,0 or 44301,6 in scheme D for blue-blue excitation scheme (not given 
in the picture; in accordance with [9]). On top of the highest transition strength among the considered UV or blue first excitation steps, the mentioned second steps have also highest relative intensity of ion count rate among all transitions studied in the respective excitation scheme.

As schemes A, B, and C involve different values of $1 / 2,3 / 2$, and $5 / 2$ for the $J$ number of the first excited level, there is also a possibility to determine unknown $J_{A I}$ values for the auto-ionizing levels in the scan range. This analysis, together with a determination of absolute ionization efficiencies is in progress and will be published separately.

\section{Conclusion}

Spectroscopic investigations in different two-step resonant ionization schemes were realized for lutetium exclusively using Titanium:sapphire laser radiation. The obtained data is used to identify an appropriate, highly efficient and highly selective ionization scheme for lutetium atoms to be used at the dedicated CERN-MEDICIS facility. These studies serve as development steps towards the implementation of a suitable RILIS laser system. In the continuation of this work, spectroscopic research will be undertaken on terbium and neodymium atoms, whose radioisotopes are expected to represent radiopharmaceuticals with high potential of application in various fields of nuclear medicine. Quantification of the ionization efficiency in the most promising excitation schemes are presently under way at the $30 \mathrm{keV}$ high transmission RISIKO off-line mass separator at the University of Mainz, which most ideally simulates the experimental conditions which will be experienced at the CERN-MEDICIS mass separator

\section{Acknowledgment}

This research project has been supported by a Marie Skłodowska-Curie Innovative Training Network Fellowship of the European Commission's Horizon 2020 Programme under contract number 642889 MEDICISPROMED.

\section{References}

1. dos Santos Augusto R. M. et al.: CERN-MEDICIS (Medical Isotopes Collected from ISOLDE): a new facility. Applied Sciences, 4, 265 (2014)

2. Letokhov V. S.: Laser photoionization spectroscopy. Academic Press, Orlando: 353 pages (1987)

3. Wendt K. et al.: Laser resonance ionization for efficient and selective ionization of rare species. Nuclear Instruments and methods in Physics Research B, 204, 325 (2003) 
4. Tishchenko V.K. et al.: Radiopharmaceuticals based on polyaminophosphonic acids labeled with $\alpha-, \beta$-, and $\gamma$-emitting radionuclides (Review). Pharmaceutical Chemistry Journal, 49(7), 3 (2015)

5. Ljungberg $M$. et al.: MIRD Pamphlet No. 26: Joint EANM/MIRD guidelines for quantitative 177Lu SPECT applied for dosimetry of radiopharmaceutical therapy. Journal of Nuclear Medicine, 57, 151 (2016)

6. Bekov G.I., Vidolova-Angelova E.P., Optimal scheme for multistage photoionization of lutetium atoms by laser radiation. Soviet Journal of Quantum Electronics, 11(1), 137 (1981)

7. Miller C. M., Nogar N. S.: Autoionizing and high-lying Rydberg states of lutetium atoms. AIP Conference Proceedings, 90, 90 (1982)

8. Krustev Th. B. et al.: Determination of traces of lutetium in geological samples by resonance ionization spectroscopy. Journal of Analytical Atomic Spectrometry, 8, 1029 (1993)

9. $\mathrm{Xu} \mathrm{C}$. B. et al.: The study of autoionizing states of lutetium atoms by resonance ionization spectroscopy. Journal of Physics B: Atomic, Molecular and Optical Physic, 26, 2821 (1993)

10. D'yachkov A. B. et al.: Selective photoionisation of lutetium isotopes. Quantum Electronics, 42(10), 953 (2012)

11. D'yachkov A. B. et al.: Photoionization spectroscopy for laser extraction of the radioactive isotope Lu-177. Applied Physics B: Lasers and Optics, 121, 425 (2015)

12. Naubereit P. et al.: Resonance ionization spectroscopy of sodium Rydberg levels using difference frequency generation of high-repetitionrate pulsed Ti:Sapphire lasers. Physical Review A, 93(5), 052518 (2016)

13. Geppert Ch.: Laser systems for on-line laser ion sources. Nuclear Instruments and methods in Physics Research B, 266, 4354 (2008)

14. Kurucz R. L., Bell B.: Atomic Line Data, Smithsonian Astrophysical Observatory, Cambridge, MA, 1995, Kurucz CD-ROM No. 23.

15. Ralchenko Yu., Kramida A. E, Reader J., and NIST ASD Team: NIST Atomic Spectra Database (version 5), [Online]. Available: https://www.nist.gov/pml/atomic-spectra-database [2016, September]. National Institute of Standards and Technology, Gaithersburg, MD.

16. https://www.alfa.com/de/catalog/089886/ 